Article

\title{
Expression of Kir2.1 Inward Rectifying Potassium Channels in Optic Nerve Glia: Evidence for Heteromeric Association with Kir4.1 and Kir5.1
}

\author{
Csilla Brasko ${ }^{1,2}$ and Arthur M. Butt ${ }^{1, *(1)}$ \\ 1 Institute of Biology and Biomedical Sciences, School of Pharmacy and Biomedical Sciences, University of \\ Portsmouth, St Michael's Building, White Swan Road, Portsmouth PO1 2DT, UK; csilla.brasko@yahoo.co.uk \\ 2 Department of Physiology, University of Szeged, Szeged Hungary Közép fasor 52, 6726 Szeged, Hungary \\ * Correspondence: arthur.butt@port.ac.uk
}

Received: 25 May 2018; Accepted: 4 July 2018; Published: 10 July 2018

\begin{abstract}
Inward rectifying potassium (Kir) channels comprise a large family with diverse biophysical properties. A predominant feature of central nervous system (CNS) glia is their expression of Kir4.1, which as homomers are weakly rectifying channels, but form strongly rectifying channels as heteromers with Kir2.1. However, the extent of Kir2.1 expression and their association with Kir4.1 in glia throughout the CNS is unclear. We have examined this in astrocytes and oligodendrocytes of the mouse optic nerve, a typical CNS white matter tract. Western blot and immunocytochemistry demonstrates that optic nerve astrocytes and oligodendrocytes express Kir2.1 and that it co-localises with Kir4.1. Co-immunoprecipitation analysis provided further evidence that Kir2.1 associate with Kir4.1 and, moreover, Kir2.1 expression was significantly reduced in optic nerves and brains from Kir4.1 knock-out mice. In addition, optic nerve glia express Kir5.1, which may associate with Kir2.1 to form silent channels. Immunocytochemical and co-immunoprecipitation analyses indicate that Kir2.1 associate with Kir5.1 in optic nerve glia, but not in the brain. The results provide evidence that astrocytes and oligodendrocytes may express heteromeric Kir2.1/Kir4.1 and Kir2.1/Kir5.1 channels, together with homomeric Kir2.1 and Kir4.1 channels. In astrocytes, expression of multiple Kir channels is the biophysical substrate for the uptake and redistribution of $\mathrm{K}^{+}$released during neuronal electrical activity known as 'potassium spatial buffering'. Our findings suggest a similar potential role for the diverse Kir channels expressed by oligodendrocytes, which by way of their myelin sheaths are intimately associated with the sites of action potential propagation and axonal $\mathrm{K}^{+}$release.
\end{abstract}

Keywords: inward rectifying potassium channel; glia; astrocyte; oligodendrocyte; white matter; potassium regulation

\section{Introduction}

Inward rectifying potassium (Kir) channels comprise of several well-defined subfamilies, including weakly rectifying Kir4.1 channels and strongly voltage-dependent Kir2.1 channels, which play key roles in setting the membrane potential in central nervous system (CNS) glia and regulating their key functions [1]. In particular, the Kir4.1 subtype plays a central role in the astrocyte function of potassium spatial buffering, which is essential for maintaining extracellular $\left[\mathrm{K}^{+}\right]$in the face of continuous $\mathrm{K}^{+}$release from electrically active neurons [2]. Oligodendrocytes are the myelin-forming cells of the CNS and also express Kir4.1 [3-5], although expression appears to be heterogeneous [3,6], and is most highly expressed in certain white matter regions, including the optic nerve [4,5]; genomic analysis by the Barres group confirms that Kir4.1 (Kcnj10) is expressed throughout the oligodendroglial lineage, although at a lower level than in astrocytes (https:/ / web.stanford.edu/group/barres_lab / cgi- 
bin/igv_cgi_2.py?lname=Kcnj10). Astrocytes and oligodendrocytes have also been shown to express strongly rectifying Kir2.1 [7-10], although in situ hybridization analyses failed to detect significant glial expression of Kir2.1 in rat brain [11], consistent with genomic analyses that, overall, Kir2.1 (Kcnj2) is expressed by astrocytes and myelinating oligodendrocytes to a much lesser extent than Kir4.1 (https:/ / web.stanford.edu/group/barres_lab/cgi-bin/igv_cgi_2.py?lname=kcnj2). In Müller glia of the retina, Kir2.1 expression is highly localized in processes that contact neurons, and their strong rectifying properties indicate they have a specific role in $\mathrm{K}^{+}$uptake during neuronal activity [12]. In hippocampal astrocytes, Kir2.1 are dramatically increased by kainic acid-induced seizure, suggesting a key role in protecting against $\mathrm{K}^{+}$accumulation caused by neuronal hyperexcitability [8].

The functional diversity of Kir is increased by the formation of heteromeric channels [1,13]. Kir2.1 and Kir4.1 are co-localized in Müller glia [12], and changes in Kir2.1 and Kir4.1 during pathophysiology suggest common mechanisms of regulation and co-assembly $[8,14]$. In addition, multiple lines of evidence indicate widespread astroglial expression of Kir5.1 [12,15-21], which on their own do not form functional channels, but by co-assembly with Kir4.1 form highly $\mathrm{pH}$ sensitive channels $[22,23]$ that are important for astrocyte-driven central chemoreception [18]. Recently, we also provided evidence that oligodendrocytes express Kir4.1/Kir5.1 channels [5]; genomic analysis indicates Kir5.1 (Kcnj16) is expressed at similar levels as Kir2.1 (Kcnj2) and more highly in immature oligodendrocytes than myelinating oligodendrocytes (https:/ / web.stanford.edu/group/barres_lab / cgi-bin/igv_cgi_2.py? lname=kcnj2). Here, we have examined the expression of Kir2.1 and its association with Kir4.1 and Kir5.1 in white matter astrocytes and oligodendrocytes of the mouse optic nerve. The results show that oligodendrocytes, like astrocytes, express Kir2.1, although to a far lesser extent than Kir4.1, and co-immunolabeling and co-immunoprecipitation analyses support the possibility that Kir2.1 may co-assemble with Kir4.1 and Kir5.1. The distinct properties of homomeric Kir2.1 and heteromeric Kir2.1/Kir4.1 and Kir2.1/Kir5.1 channels provide a biophysical basis for efficient $\mathrm{K}^{+}$spatial buffering in oligodendrocytes, as well as astrocytes, during the large shifts in $\mathrm{K}^{+}$to which they are exposed during action potential propagation.

\section{Materials and Methods}

\subsection{Animals and Optic Nerve Explant Cultures}

The mice used were of the C57 strain, or transgenic mice in which the oligodendroglial myelin gene proteolipid protein (PLP) drives expression of Discosoma sp. red fluorescent protein (DsRed) (PLP-DsRed; from Prof. Frank Kirchhoff, University of Saarland, Homburg, Germany), or Kir4.1 knock-out (KO) mice (from Prof. Christian Steinhäuser, University of Bonn, Germany; originally generated by Kofuji et al.) [24]. Mice were killed humanely by cervical dislocation, approved by the Home Office of the United Kingdom under the Animals (Scientific Procedures) Act, 1986, licence number $70 / 7733$.

\subsection{Optic Nerve Explant Cultures}

Mice aged postnatal day $(\mathrm{P}) 7-11$ were used to prepare mixed glial cultures from optic nerve explants [25]. Optic nerves were carefully removed to dissecting media, comprised of Dulbecco's Modified Eagle Medium (DMEM) (Sigma-Aldrich, Irvine, UK), supplemented with $4 \mathrm{mM}$ L-glutamate, $10 \%$ fetal bovine serum (FBS; Invitrogen, Paisley, UK) and $0.1 \%$ gentamicin (Invitrogen). Nerves were cut into 1-2 mm fragments prior to trituration and transference onto poly-L-lysine (Sigma-Aldrich) coated coverslips and incubated at $37{ }^{\circ} \mathrm{C}$ in $5 \% \mathrm{CO}_{2}$. After $24 \mathrm{~h}$, cultures were placed in low serum $(0.5 \%)$ modified Bottenstein and Sato (B\&S) culture medium, supplemented with $10 \mathrm{ng} / \mathrm{ml}$ recombinant human platelet-derived growth factor (PDGF)-AA (R\&D Systems, Abingdon, UK) and $0.1 \%$ gentamicin. After 3-4 days in vitro (DIV), the medium was changed to B\&S supplemented with $0.5 \mathrm{mM}$ dibutyryl cAMP for up to $10 \mathrm{DIV}$, to promote oligodendrocyte differentiation, then B\&S media with $0.1 \%$ gentamicin for up to 15 DIV, changing media every $3-5$ days. 


\subsection{Immunocytochemistry}

After 11-15 DIV, optic nerve explant cultures were fixed in 1\% paraformaldehyde in phosphate buffered saline (PBS, pH 7.4) for $10 \mathrm{~min}$ at room temperature (RT). Following washes in PBS, coverslips were incubated in blocking solution (5\% normal goat serum, NGS, in PBS) for $1 \mathrm{~h}$ at RT and then permeabilized in 5\% NGS in PBS with $0.2 \%$ Triton for 15-30 min, prior to incubation overnight at $4{ }^{\circ} \mathrm{C}$ in primary antibodies diluted in PBS (chicken anti-GFAP, 1:500, Chemicon, UK; in-house rabbit anti-Kir4.1, 1:400 [4,5]; rabbit anti-Kir5.1, 1:300, Alomone Labs Ltd., Jerusalem, Israel; goat anti-Kir2.1, 1:100, (Santa Cruz Biotechnology, TX, USA). Following washes, coverslips were incubated for $1 \mathrm{~h}$ at RT in secondary antibodies diluted in PBS (goat-anti rabbit Alexafluor 488, 1:400, Molecular Probes; donkey anti-goat 488, Molecular Probes; donkey anti-chicken Alexafluor 568, Molecular Probes UK; donkey anti-chicken Dylight 405, 1:400, Stratech, Ely, UK; donkey anti-rabbit Dylight 647, 1:200, Stratech). Finally, coverslips were washed and mounted on slides with Vectasheild ${ }^{\circledR}$ (VectorLabs, Peterborough, UK). Negative controls were carried out for all antibodies, using pre-incubation with appropriate antigens for Kir2.1 and Kir5.1, according to manufacturer's recommendations, and for Kir4.1 specificity was confirmed using tissue from Kir4.1 knock-out mice.

\subsection{Image Capture and Analysis}

Immunolabelled coverslips were imaged on a Zeiss LSM 710 confocal microscope (Zeiss, Oberkochen, Germany), using the $\times 40, \times 63$, or $\times 100$ oil immersion objectives. Fluorescence was detected using excitation wavelengths of $488 \mathrm{~nm}$ (green), $568 \mathrm{~nm}$ (red), $633 \mathrm{~nm}$ (far red) and $405 \mathrm{~nm}$ (blue), with argon, $\mathrm{HeNe} 1$ and diode lasers, respectively. Images were captured using optimal settings for pinhole diameter $(0.13-0.3 \mu \mathrm{m})$, detector gain and offset acquisition to detect the positive signal with minimal background, and multi-track capture of separate channels was used to prevent 'bleed'. Z-stacks comprising 4-15 optical sections (1024 $\times 1024$ pixels) were used to generate three-dimensional images (voxel size 43-76 nm xy/76-283 nm z), using Zen 2009 Light software version $\mathrm{x}$ (Zeiss). Identical settings were used to image negative controls. Co-localization analysis was carried out using Volocity 6.1 software (PerkinElmer, USA), by measuring signal overlap from the degree of separation between pixels from the red and green channels in single $z$-sections, as described by Barlow et al. and used in our previous studies [5,26]. Images were thresholded to separate the positive signal (positive immunolabelling) from background, by measuring the background intensity value for each channel in negative control sections and setting the threshold as the mean background intensity plus three standard deviations (averaged from a minimum of six images). The thresholded Pearson's correlation coefficient (PCC) was used to measure the degree of overlap between the red and green channels, to generate a co-localization channel in three-dimensions and the number of voxels in which the two channels overlap with the same intensity.

\subsection{Western Blot and Co-Immunoprecipitation}

Whole brains or optic nerves dissected from young adult mice aged 20-30 days old were homogenized in buffer containing $12.5 \mathrm{mM} \mathrm{NaCl}, \mathrm{pH} \sim 8,2 \mathrm{mM}$ Tris/ $\mathrm{HCl}, 0.2 \mathrm{mM}$ phenyl-methyl sulphonyl fluoride (PMSF), distilled water and $1 \times$ complete mini protease inhibitor cocktail (Roche, Basel, Switzerland), on ice. Samples were centrifuged at $12,000 \mathrm{rpm}$ for $5 \mathrm{~min}$ at $4{ }^{\circ} \mathrm{C}$ and the aspirated supernatant was placed in a fresh tube on ice. Quantification of protein concentration was carried out with bicinchoninic acid assay (Sigma-Aldrich) with a standard bovine serum albumin (BSA) concentration curve and ultraviolet (UV) spectrophotometer absorbance readings at $550 \mathrm{~nm}$. Samples were used either as whole lysates or subcellular fractions were prepared, as described below.

Western blot was performed on whole tissue lysates, prepared as above, or subcellular fractions prepared, as detailed previously [5]. In brief, whole brains and optic nerves of wild type and Kir4.1 $\mathrm{KO}$ animals were homogenized in subcellular fractionation buffer ( $250 \mathrm{mM}$ sucrose, $20 \mathrm{mM}$ HEPES, $10 \mathrm{mM} \mathrm{KCl}, 1.5 \mathrm{mM} \mathrm{MgCl} 2,1 \mathrm{mM}$ ethylenediaminetetraacetic acid (EDTA), $1 \mathrm{mM}$ EGTA, 1 DTT 
and $1 \times$ protease inhibitor cocktail), then centrifuged at $4{ }^{\circ} \mathrm{C}$ with $750 \times g$ for $10 \mathrm{~min}$ to remove the nuclear fraction; the supernatant was then placed in a new centrifuge tube and centrifuged with $10,000 \times g$ at $4{ }^{\circ} \mathrm{C}$ two times to remove the mitochondrial fraction and the supernatant was placed in an ultracentrifuge tube and centrifuged with $40,000 \times g$ at $4{ }^{\circ} \mathrm{C}$ for $1 \mathrm{~h}$ to leave the pellet containing the crude plasma membrane fraction, which was re-suspended in $400 \mu \mathrm{L}$ fractionation buffer using a $25 \times g$ needle and centrifuged at $40,000 \times g$ at $4{ }^{\circ} \mathrm{C}$ for $45 \mathrm{~min}$ and then the pellet was re-suspended in lysis buffer. Subsequently, total lysates and subcellular fractions were treated the same and prepared for sodium dodecyl sulfate polyacrylamide gel electrophoresis (SDS-PAGE); samples were mixed with Laemmli sample buffer and heated at $95^{\circ} \mathrm{C}$ for $5 \mathrm{~min}$ with $\beta$-mercaptoethanol and loaded for $10 \%$ acrylamide SDS-PAGE, submerged in electrophoresis buffer $\mathrm{pH} \sim 8$ containing of $25 \mathrm{mM}$ Tris base $190 \mathrm{mM}$ glycine $0.1 \%$. Proteins were then electrophoretically transferred to a polyvinyllidene difluoride membrane (Amersham) that had been incubated for $1 \mathrm{~h}$ in blocking solution of $5 \%$ dried milk in Tris buffered saline (TBS; $150 \mathrm{mM} \mathrm{NaCl} 10 \mathrm{mM}$ Tris pH 7.4) with $0.05 \%$ Tween 20, and incubated in primary overnight at $4{ }^{\circ} \mathrm{C}$ in antibodies diluted in $5 \%$ dried milk and Tris buffered saline: rabbit anti-Kir4.1, 1:1000 [4], rabbit anti-Kir2.1, 1:300 (Alomone Labs Ltd.), goat anti-Kir2.1, 1:300 (Santa Cruz Biotechnology). Following washes, incubation in horseradish peroxidase-conjugated secondary antibodies diluted in 5\% dried milk and Tris buffered saline was carried out for $1 \mathrm{~h}$ at RT: rabbit anti-goat, 1:3000 (Dako,), goat anti-mouse, 1:10,000 (Dako), swine anti-rabbit 1:2000 (Dako). Extensive washing of the membranes in TBS with ice cold $0.05 \%$ Tween 20 was performed after each incubation and immunocomplexes were visualized using an enhanced chemiluminescence method (Amersham).

Co-immunoprecipitation analysis was performed on whole tissue lysates prepared as above, using $\mu$ MACS $^{\mathrm{TM}}$ Protein A/G MicroBead kits (Miltenyi Biotec, Bergisch Gladbach, Germany) according to the manufacturers recommendations [25]. In brief, lysates were centrifuged at $4{ }^{\circ} \mathrm{C}$ with $10,000 \times g$ 2 times to remove cell debris and $3 \mu \mathrm{g}$ polyclonal or $2 \mu \mathrm{g}$ monoclonal antibody was added to the proteins and incubated overnight at $4{ }^{\circ} \mathrm{C}$; for negative controls, proteins were mixed with pre-absorbed antibodies for Kir2.1 and Kir5.1 and Kir4.1 KO tissue was used to confirm the specificity of Kir4.1 antibodies. Then, Protein G MicroBeads were added to the samples to form magnetically labeled immune complexes $(50 \mu \mathrm{L}$ for monoclonal antibody or $100 \mu \mathrm{L}$ for polyclonal antibody was used for the precipation) for $30 \mathrm{~min}$ on ice. Homogenates were then run through a $\mu$ Column placed in the magnetic field of the $\mu \mathrm{MACS}{ }^{\mathrm{TM}}$ separator and the immune complex was eluted by applying pre-heated $\left(95^{\circ} \mathrm{C}\right) 1 \times$ Laemelli sample buffer to the column. The drop on the column tip containing the eluted immunoprecipitate was collected and stored on ice or at $-80^{\circ} \mathrm{C}$ until it was analyzed by SDS-PAGE.

\section{Results}

Optic nerve explants cultures from P7-11 mice [5,25] were examined after 15 DIV to determine the expression of Kir2.1 in oligodendrocytes, identified by their expression of the PLP-DsRed reporter (Figure 1A), and astrocytes, identified by co-immunostaining for glial fibrillary acidic protein (GFAP) (Figure 1B). Immunostaining for Kir2.1 was punctate (Figure 1Ai,Bi) and in oligodendrocytes was localized to the tips of their processes (Figure 1Aiii), whereas in astrocytes immunostaining was localized to the cell somata and primary processes (Figure 1Biii). No immunostaining was observed in negative controls pre-incubated in the blocking peptides (Figure 1C) and as a further test of the specificity, in the absence of Kir2.1 knock-out tissue, we used two separate antibodies [27], namely a rabbit polyclonal anti-Kir2.1 antibody (Alomone Labs Ltd.) raised against amino acid sequence 393-411 (Accession AAI37842.1), and a goat polyclonal anti-Kir2.1 antibody (C-20, Santa Cruz Biotechnology, Dallas, TX, USA) raised against amino acid sequence 378-427 (Accession P63252); immunostaining was equivalent for the two antibodies in both oligodendrocytes and astrocytes (not illustrated). Furthermore, Western blot analysis of Kir2.1 in brain and optic nerve lysates using either antibody detected a predicted band at $\sim 45 \mathrm{kDa}$, the molecular weight (MW) of Kir2.1, with an additional band at $\sim 60 \mathrm{kDa}$, which is considered to be the fully glycosylated plasma membrane bound Kir2.1 protein (Figure 1D). In support of this, $60 \mathrm{kDa}$ Kir2.1 was enriched in the optic nerve plasma membrane 
fraction (Figure 1D), which also demonstrates that Kir2.1 is localized to glial cell membranes, where it can form functional channels.
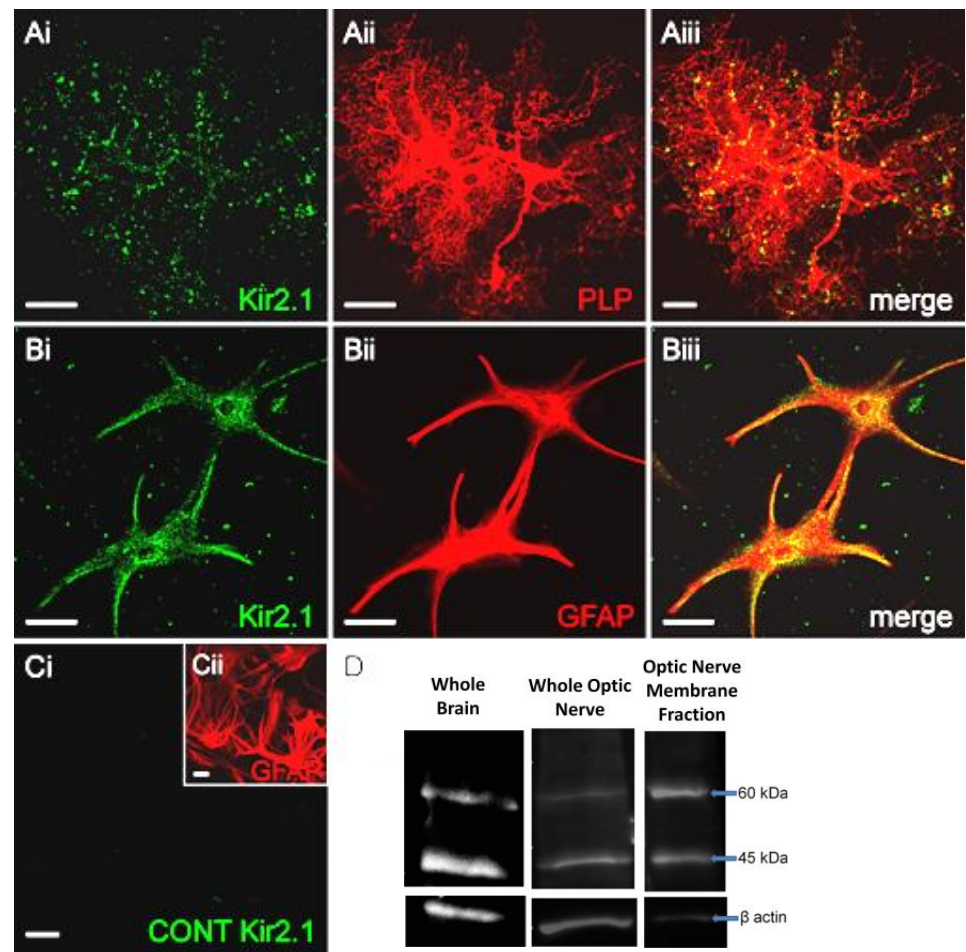

Figure 1. Expression of Kir2.1 (inward rectifying potassium channel 2.1) in optic nerve glia. (A-C) Optic nerves from P7-11 mice were maintained in explant culture for 15 days in vitro (DIV) and immunolabelled for Kir2.1 ((Ai,Bi), green) in oligodendrocytes, identified by expression of the proteolipid protein, Discosoma sp. red fluorescent protein (PLP-DsRed) reporter ((Aii), red), and astrocytes, identified by glial fibrillary acidic protein (GFAP) immunolabelling ((Bii), red); merged images show co-localisation ((Aiii,Biii), co-expression appears yellow) and no signal was detected in negative controls (C). Scale bars $=20 \mu \mathrm{m}$. (D) Western blot analyses for Kir2.1 in lysates of whole brain (left-hand panel) and optic nerve (middle panel), together with optic nerve plasma membrane fraction (right-hand panel), from young adult mice aged P2-30; bands were detected at $\sim 45 \mathrm{kDa}$ as predicted from the molecular weight of the Kir2.1 protein, together with a band at $\sim 60 \mathrm{kDa}$ that was more enriched in the plasma membrane fraction and is considered to be the fully glycosylated Kir2.1 protein.

Next, we used double immunofluorescence in optic nerve explants cultures to demonstrate that Kir2.1 are co-expressed with Kir4.1 in oligodendrocytes (Figure 2Ai-Aiii) and astrocytes (Figure 2Bi-Biii); immunostaining was not observed in negative controls (Figure 2Bix,Bx). To determine the extent of co-localization of Kir2.1 and Kir4.1, we used the technique of Barlow and colleagues [26], as previously described $[5,28]$, to generate a co-localization channel of the voxels in which fluorescence is at equal intensity from the green (Kir2.1) and red (Kir4.1) channels (Figure 2Aiv,Biv). The results demonstrate a high degree of co-localization in oligodendrocytes (Figure 2Aiv-Avi) and astrocytes (Figure 2Biv-Bvi), predominantly on the processes of both cell types (Figure 2Avii-Aix, Bvii-Bviii). Quantification demonstrated greater overall expression of Kir2.1 in astrocytes compared to oligodendrocytes, and markedly less than Kir4.1 in both cell types (Figure 2C,D); 50\% of immunostaining for Kir2.1 was co-localized with Kir4.1 in both cell types (astrocytes: 24,839 $\pm 11,445$ Kir2.1 $1^{+}$Kir4.1 $1^{+}$voxels per cell, compared to $47,152 \pm 22,079$ Kir2.1 $1^{+}$voxels and 149,541 $\pm 58,462$ Kir4.1 $1^{+}$voxels per cell $(n=13)$; oligodendrocytes: $32,755 \pm 15,080 \mathrm{Kir} 4.1+/$ Kir2.1+ voxels per cell, compared to $68,294 \pm 34,663 \mathrm{Kir} 2.1^{+}$voxels and 189,959 $\pm 100,143 \mathrm{Kir} 4.1+$ voxels per cell $(n=12)$. Co-immunoprecipitation analyses of total lysates from brain and optic nerve of P20-30 
mice provides further evidence that Kir2.1 associate with Kir4.1 (Figure 2F-H). The $45 \mathrm{kDa}$ protein immunoprecipitated with the Kir2.1 antibody (Figure 2F) co-immunoprecipated with Kir4.1 antibody specifically at $\sim 42 \mathrm{kDa}$, the MW for Kir4.1 (Figure 2G), and the reverse co-immunopreciptation gave equivalent results (Figure $2 \mathrm{H}$ ); this was confirmed in four experiments, all unequivocally demonstrating the same result, and no co-immunoprecipitation was detected in negative controls.
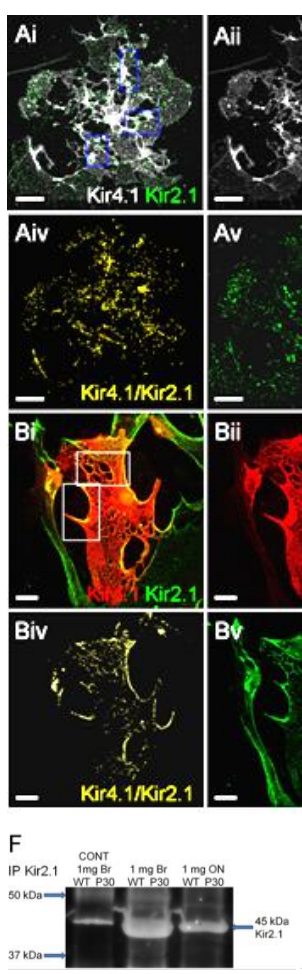
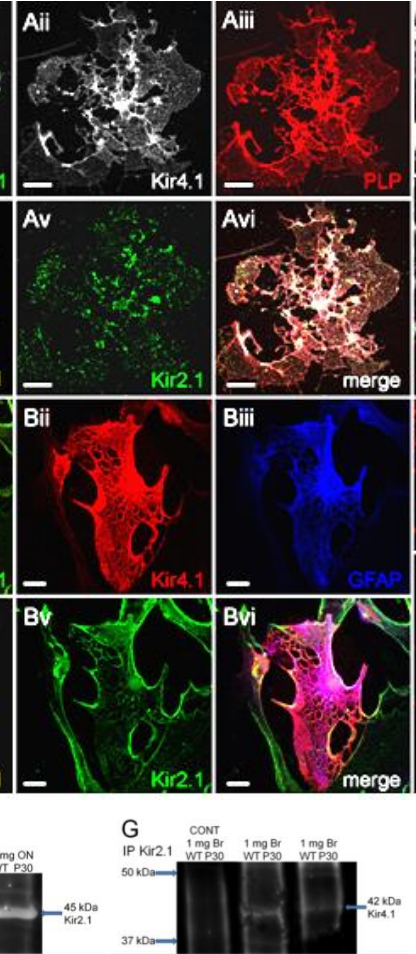
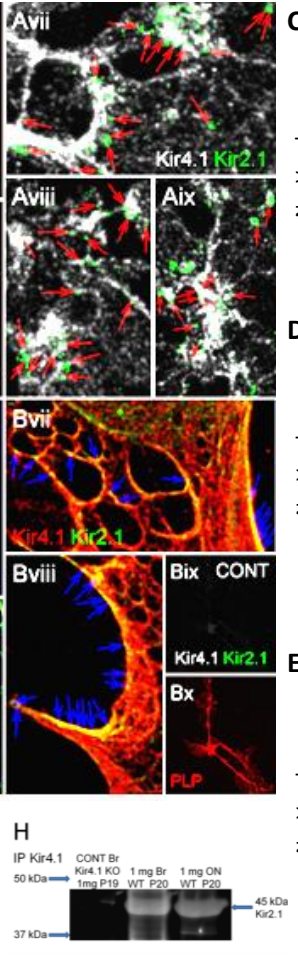

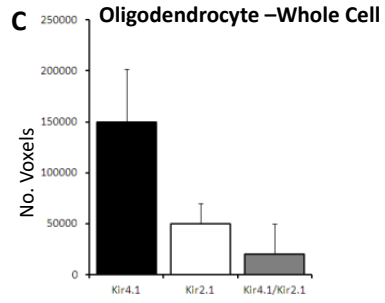

D
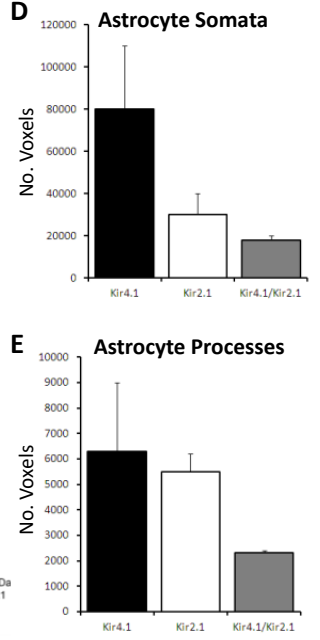

Figure 2. Co-expression of Kir2.1 and Kir4.1 in optic nerve glia. (A,B) Double immunofluorescence labeling in optic nerve glia isolated from P11 optic nerve cultured for 11 DIV: (A) Kir4.1 (far red, appears white) and Kir2.1 (green) in oligodendrocytes identified by expression of the PLP-DsRed reporter (red); (B) Kir4.1 (red) and Kir2.1 (green) in astrocytes identified by immunolabelling for GFAP (blue). Co-localization channels for Kir2.1/Kir4.1 are illustrated (Aiv,Biv), together with high magnification merged images indicating co-localization in processes (Avii-Aix,Bvii-Bvii); no signal was detected in negative controls (Bix-Bx). Scale bars $=20 \mu \mathrm{m}$. (C-E) Kir4.1/Kir2.1 co-localization analysis in oligodendrocytes $(\mathbf{C})$, astrocytes $(\mathbf{D})$ and astrocyte processes $(\mathbf{E})$; data are means + standard error of the mean (SEM), $n=13)$. (F-H) Co-immunoprecipitation analysis of Kir2.1 and Kir4.1 from lysates of whole brain and optic nerve from mice aged P20-30 detected predicted bands at $45 \mathrm{kDa}$ for the anti-Kir2.1 antibody (F) and at $\sim 42 \mathrm{kDa}$ when co-immunprecipated with anti-Kir4.1 antibody (G), with equivalent results when immunoprecipated first with the anti-Kir4.1 antibody and then co-immunoprecipated with the anti-Kir2.1 antibody (H); no bands were detected in negative controls.

The results indicate that Kir4.1 and Kir2.1 subunits co-assemble in optic nerve glia and to further investigate this, we determined whether Kir2.1 expression is altered in Kir4.1 knock-out mice, in which we and others have previously demonstrated Kir4.1 to be absent in the brain and optic nerve $[5,29,30]$. Comparison of whole lysates from brain and optic nerve in controls (Figure 3A) and Kir4.1 KO mice (Figure 3B) demonstrates a significant decrease in expression of the $45 \mathrm{kDa}$ Kir2.1 protein in the absence of Kir4.1 (unpaired $t$-tests, $p$ values indicated on graph). Conversely, the $60 \mathrm{kDa}$ glycosalted band in the plasma membrane fraction was significantly increased in Kir4.1 KO brain to $1.47 \pm 0.52$ compared to $0.95 \pm 0.098$ in wild-type (Figure 3B; $p<0.01$, unpaired $t$-test). These data suggest that mechanisms of regulation for Kir2.1 and Kir4.1 are closely inter-related. 


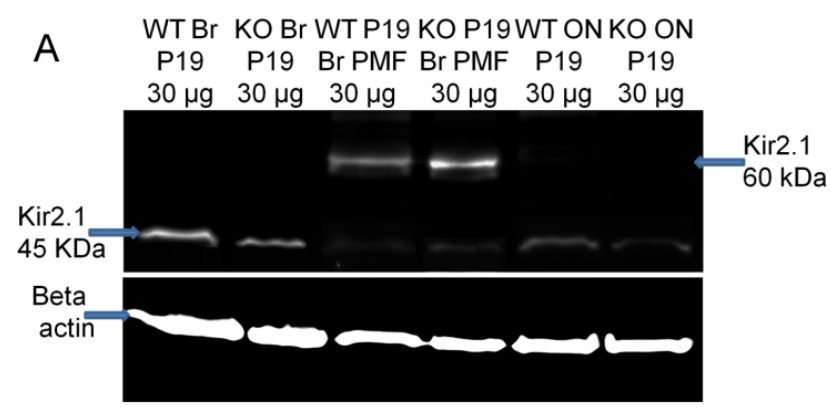

Kir2.1 protein levels in the whole brain, brain B plasma membrane fraction and optic nerves of the wild type and Kir4.1 knockout mice

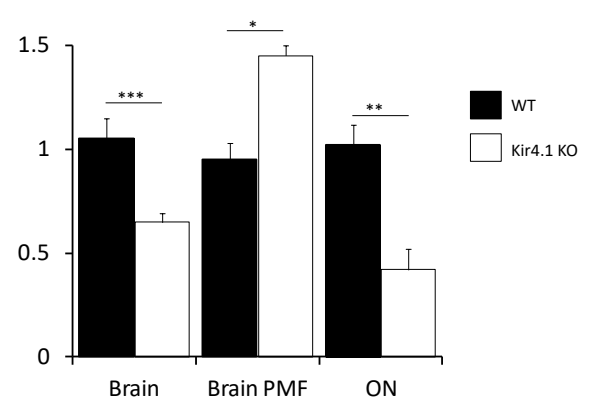

Figure 3. Kir2.1 protein levels are altered in the absence of Kir4.1. (A) Western blot analysis of Kir2.1 in total brain lysate (lanes 1,2), brain plasma membrane fraction (lanes 3,4) and total optic nerve lysate (lanes 5, 6), from P19 wild-type (WT) and (lanes 1, 3, 5) and Kir4.1 knock-out (KO) mice (lanes 2, 4, 6); predicted bands were detected at $\sim 45 \mathrm{kDa}$ in whole lysates (lanes 1, 2, 5, 6) and a $\sim 60 \mathrm{kDa}$ band for the fully glycosylated plasmalemmal channel enriched in the plasma membrane fraction (PMF) (lanes 3 , 4). (B) Mean ( $\pm \mathrm{SEM}, n=3$ ) density of the $45 \mathrm{kDa}$ band for brain and optic nerve, and $60 \mathrm{kDa}$ band for brain plasma membrane fraction, normalized against $\beta$-actin; ${ }^{*} p<0.05,{ }^{* *} p<0.01,{ }^{* * *} p<0.001$, unpaired $t$-tests.

Finally, we investigated the possibility of an association between Kir2.1 and Kir5.1, since it has been reported they may form heteromers [31] and we recently showed optic nerve glia express Kir5.1 that did not entirely co-localize with Kir4.1 [5], with which Kir5.1 preferentially form heteromers [23]. Double immunofluorescence labeling of optic nerve explant cultures demonstrates Kir2.1 and Kir5.1 are co-expressed in oligodendrocytes (Figure 4A) and astrocytes (Figure 4B), with a high degree of co-localization in both cell types (Figure 4Aiv-Avi,Biv-Bvi); $40 \%$ of Kir2.1 voxels co-localised with Kir5.1 (Figure 4C,D), whereas conversely only 25\% of Kir5.1 were co-localized with Kir2.1 in oligodendrocytes and $16 \%$ in astrocytes, consistent with Kir5.1 mainly associating with Kir4.1 [5,23]. Immunoprecipitation of whole brain and optic nerve lysates from P23-27 mice with anti-Kir5.1 antibody identified a specific $\sim 48 \mathrm{kDa}$ protein, the MW of Kir5.1, and co-immunoprecipitation with anti-Kir2.1 antibody identified the predicted $45 \mathrm{kDa}$ protein in the optic nerve, but in the brain (Figure $4 \mathrm{~F}$ ); results were confirmed in four experiments, all unequivocally demonstrating the same result, and no bands were detected in negative controls. The data suggest Kir2.1 may co-assemble with Kir5.1 in optic nerve glia. 


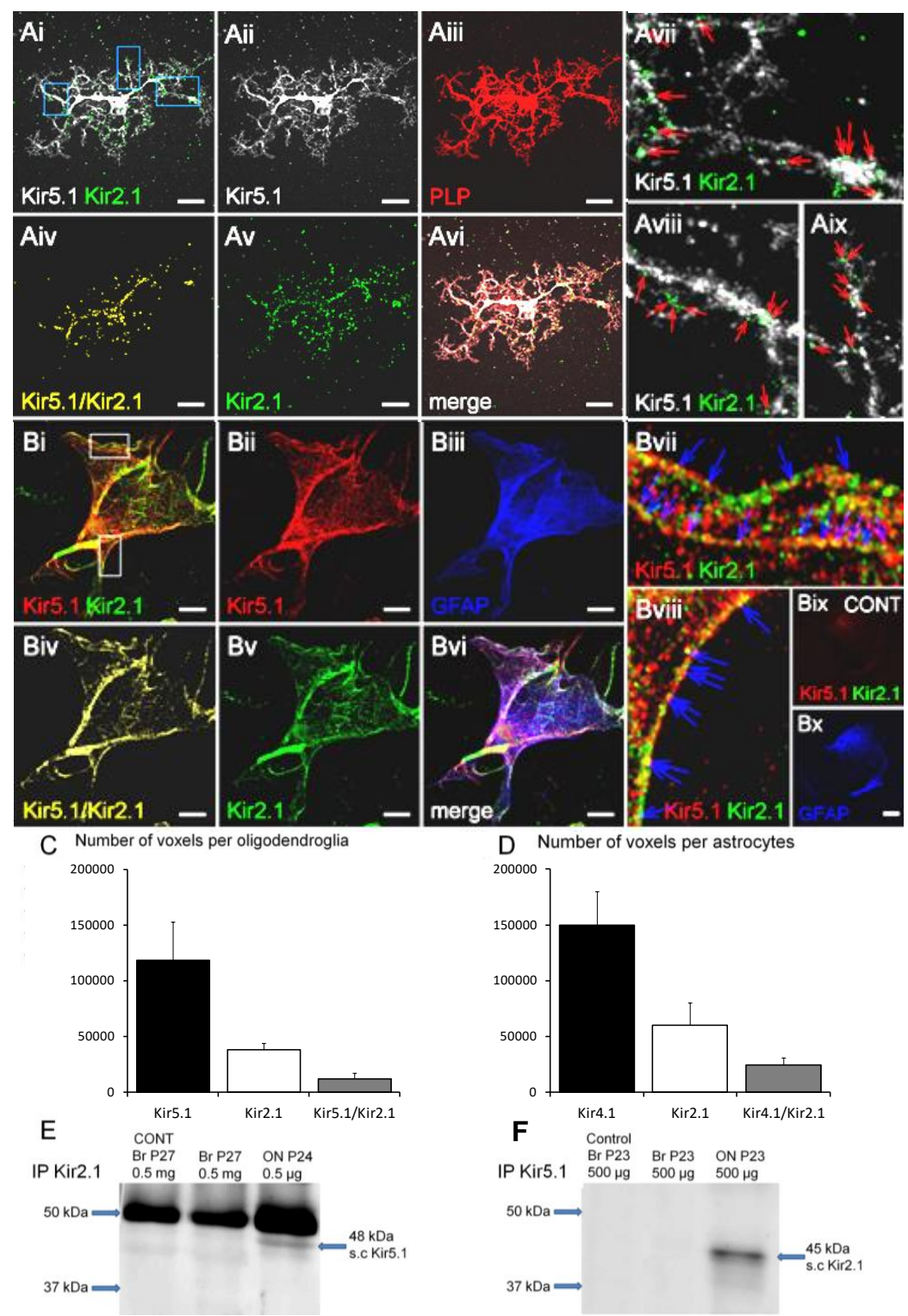

Figure 4. Co-expression of Kir2.1 and Kir5.1 in optic nerve glia. (A,B) Double immunofluorescence labeling in optic nerve glia from P11 optic nerve cultured for 11 DIV: (A) Kir5.1 (far red, appears white) and Kir2.1 (green) in oligodendrocytes identified by expression of the PLP-DsRed reporter (red); (B) Kir5.1 (red) and Kir2.1 (green) in astrocytes identified by immunolabelling for GFAP (blue). Co-localisation channels for Kir5.1/Kir2.1 are illustrated (Aiv,Biv), together with high magnification merged images indicating co-localization in processes (Avii-Aix,Bvii-Bvii); no signal was detected in negative controls (Bix-Bx). Scale bars $=20 \mu \mathrm{m}$. (C-E) Kir5.1/Kir2.1 co-localization analysis in oligodendrocytes $(\mathbf{C})$ and astrocytes $(\mathbf{D})$; data are means \pm SEM, $n=13)$. (E,F) Co-immunoprecipitation analysis of Kir5.1 with Kir2.1 from lysates of whole brain and optic nerve from mice aged P20-30 detected predicted bands at $\sim 48 \mathrm{kDa}$ for the anti-Kir5.1 antibody (E) and at $\sim 45 \mathrm{kDa}$ when co-immunoprecipated with anti-Kir2.1 antibody in optic nerve, but not brain $(\mathbf{G})$. No bands were detected in negative controls.

\section{Discussion}

In glia, Kir2.1 are considered to have an important role in the astroglial function of $\mathrm{K}^{+}$clearance, due to their strong rectifying properties and cellular expression on processes of Müller glia and Schwann cells that contact neurons and axons [12,32]. Here, we demonstrate that in CNS white matter 
of the mouse optic nerve, both oligodendrocytes and astrocytes express Kir2.1 and provide evidence they exist alone and in association with Kir4.1 and Kir5.1. The results support the possibility that in CNS white matter homomeric Kir2.1 and heteromeric Kir2.1/Kir4.1 and Kir2.1/Kir5.1 channels interact to play an important role in $\mathrm{K}^{+}$regulation in astrocytes and oligodendrocytes.

Expression of Kir2.1 in optic nerve glia supports studies in other CNS regions [7-10]. Compared to Kir4.1 expression, Kir2.1 immunostaining was weak, which is consistent with retinal astrocytes, where Kir2.1 expression is 12 times lower than Kir4.1 [14], and with genomic analyses that Kir2.1 (Kcnj2) is expressed by astrocytes and myelinating oligodendrocytes to a much lesser extent than Kir4.1 (Kcnj10) (https://web.stanford.edu/group/barres_lab/cgi-bin/igv_cgi_2.py?lname=kcnj2). The relatively low expression of Kir2.1 in glia may also help explain why in situ hybridization analyses failed to detect significant glial expression of Kir2.1 in rat brain [11]. Due to technical reasons, immunoblot analyses were performed on tissue from P20-30 mice and immunocytochemical analyses were performed on optic nerve explant cultures from P7-11 mice after 11-15 DIV, hence the ages were compatible. It remains a possibility that the relative expression levels of different Kir subunits may change with age, but the immunocytochemistry and immunoblot data are in accord that in the brain and optic nerve Kir2.1 is strongly associated with Kir4.1, which is almost exclusively glial in the CNS [1,6], providing strong supporting evidence that glia express Kir2.1.

It is known that Kir2.1 channels form functional heteromeric channels with Kir4.1, changing the latter from weak rectifying to strongly rectifying channels [33]. Our results support other studies showing co-localization of Kir2.1 and Kir4.1 in astrocytes and Müller glia [8,12,14], and for the first time demonstrate Kir2.1/Kir4.1 co-localization in oligodendrocytes. Expression of Kir2.1 was significantly decreased in Kir4.1 KO mice, suggesting a high degree of inter-dependence and common mechanisms of regulation and function. Moreover, plasmalemmal Kir2.1 was relatively greater in the absence of Kir4.1, suggesting a role for Kir4.1 in the cellular localization of Kir2.1. Previous studies confirmed the expression of Kir2.1 subunit in the retina from Kir4.1 KO mice, although they did not detect significant changes in the expression of Kir2.1 in Müller glia from Kir4.1 KO mice [12]. In the condition termed proliferative vitreoretinopathy that is associated with proliferative gliosis, Kir4.1 and Kir2.1 were found to be inactivated by mislocalisation in retinal Müller cells [14]. In hippocampal astrocytes, where Kir4.1 is the predominant $\mathrm{K}^{+}$channel subunit [34], the accumulation of extracellular $\mathrm{K}^{+}$caused by neuronal hyperexcitability results in the loss of the perivascular weak inward rectifying Kir4.1 channels [35] and an elevation of strong inward rectifying Kir2.1 [8]. Together, these studies demonstrate an inter-dependence of Kir4.1 and Kir2.1 expression in glial cells.

An association between Kir2.1 and Kir5.1 was also demonstrated in optic nerve glia. Co-assembly of Kir2.1 and Kir5.1 subunits has been demonstrated in Xenopus oocytes [31], but another study did not find that Kir5.1 can associate with Kir2.1 [23]. We did not find evidence that Kir2.1 associated with Kir5.1 in the brain, but only in the optic nerve. In contrast, here and in a recent study [5], evidence of Kir2.1/Kir4.1 and Kir4.1/Kir5.1 was found in both brain and optic nerve. Overall, the results are consistent with co-assembly of Kir2.1 and Kir5.1 being a special feature of optic nerve glia. In contrast, Kir2.1/Kir4.1 and Kir4.1/Kir5.1 channels appear to be widely expressed throughout the brain $[5,8,12,14-21]$ and respectively form strongly rectifying and highly $\mathrm{pH}$ sensitive channels [22,23]. Notably, co-assembly of Kir2.1 with Kir5.1 results in the formation of an electrically silent channel [31]. Thus, Kir5.1 potentially have a major regulatory role in glia, by silencing Kir2.1 and removing them from forming strongly rectifying channels, as homers or heteromers with Kir4.1, which in the absence of Kir2.1 would otherwise form weakly rectifying channels. Interestingly, we previously showed that plasmalemmal Kir5.1 persist in the absence of Kir4.1 [5] and now show the same is true for plasmalemmal Kir2.1. Since Kir5.1 cannot form functional homomeric channels, these results suggest that in the absence of Kir4.1, Kir2.1 may co-assemble with Kir5.1 to form electrically silent channels. This possibility is supported by the almost complete loss of inward rectifying potassium currents in astrocytes and oligodendrocytes upon the genetic deletion of Kir4.1 gene [29,30,36,37], despite our evidence of continued expression of strongly rectifying Kir2.1 in the absence of Kir4.1. The absence of 
co-immunoprecipitation for Kir2.1 and Kir5.1 in brain lysates, comprising mainly cortex, suggests that Kir5.1 may not have an equivalent regulatory function in cortical glia, where Kir4.1 have been shown to the dominant potassium channel, although our analyses may not be sufficiently sensitive to detect low levels of co-expression. Together, the results indicate close interactions between Kir2.1, Kir4.1 and Kir5.1 that determine their localization and function in optic nerve glia.

In summary, this study identifies expression of Kir2.1 and Kir2.1/Kir4.1 channels in oligodendrocytes as well as astrocytes, and provides the first evidence of an association between Kir2.1 and Kir5.1, which may be a special feature of optic nerve glia, although further studies are required to determine whether these may be features of white matter glia throughout the CNS. Interestingly, co-assembly of Kir2.1 with Kir4.1 forms a strongly rectifying channel, whereas co-assembly with Kir5.1 results in the formation of an electrically silent channel. Thus, the plasmalemmal expression of Kir2.1 and Kir4.1 as homomeric channels or as heteromeric channels with each other or with Kir5.1 would determine glial membrane properties and their capacity for $\mathrm{K}^{+}$uptake and provide a potential mechanism for $\mathrm{K}^{+}$spatial buffering. At sites of high neuronal activity, $\mathrm{K}^{+}$released into the extracellular space would be taken up via strongly rectifying Kir2.1 and Kir2.1/Kir4.1 channels, whereas $\mathrm{K}^{+}$would be released back into the extracellular space at sites of low $\mathrm{K}^{+}$; for example, during low neuronal activity or at blood vessels, via weakly rectifying Kir4.1 channels combined with silencing of Kir2.1 channels by their co-assembly with Kir5.1.

Author Contributions: A.M.B. and C.B. both contributed to the design and analysis of the data; experiments were performed by C.B.; the paper was written by A.M.B. and C.B.; funding was to A.M.B.

Funding: This research was funded by the BBSRC (BB/J016888) and MRC (MR/P025811/1).

Conflicts of Interest: The authors declare no conflict of interest. The founding sponsors had no role in the design of the study; in the collection, analyses, or interpretation of data; in the writing of the manuscript, and in the decision to publish the results.

\section{References}

1. Butt, A.M.; Kalsi, A. Inwardly rectifying potassium channels (Kir) in central nervous system glia: A special role for Kir4.1 in glial functions. J. Cell. Mol. Med. 2006, 10, 33-44. [CrossRef] [PubMed]

2. Kofuji, P.; Connors, N.C. Molecular substrates of potassium spatial buffering in glial cells. Mol. Neurobiol. 2003, 28, 195-208. [CrossRef]

3. Poopalasundaram, S.; Knott, C.; Shamotienko, O.G.; Foran, P.G.; Dolly, J.O.; Ghiani, C.A.; Gallo, V.; Wilkin, G.P. Glial heterogeneity in expression of the inwardly rectifying $\mathrm{K}^{+}$channel, Kir4.1, in adult rat CNS. Glia 2000, 30, 362-372. [CrossRef]

4. Kalsi, A.S.; Greenwood, K.; Wilkin, G.; Butt, A.M. Kir4.1 expression by astrocytes and oligodendrocytes in CNS white matter: A developmental study in the rat optic nerve. J. Anat. 2004, 204, 475-485. [CrossRef] [PubMed]

5. Brasko, C.; Hawkins, V.; De La Rocha, I.C.; Butt, A.M. Expression of Kir4.1 and Kir5.1 inwardly rectifying potassium channels in oligodendrocytes, the myelinating cells of the CNS. Brain Struct. Funct. 2017, 222, 41-59. [CrossRef] [PubMed]

6. Tang, X.; Taniguchi, K.; Kofuji, P. Heterogeneity of Kir4.1 channel expression in glia revealed by mouse transgenesis. Glia 2009, 57, 1706-1715. [CrossRef] [PubMed]

7. Howe, M.W.; Feig, S.L.; Osting, S.M.; Haberly, L.B. Cellular and subcellular localization of Kir2.1 subunits in neurons and glia in piriform cortex with implications for $\mathrm{K}^{+}$spatial buffering. J. Comp. Neurol. 2008, 506, 877-893. [CrossRef] [PubMed]

8. Kang, S.J.; Cho, S.H.; Park, K.; Yi, J.; Yoo, S.J.; Shin, K.S. Expression of Kir2.1 channels in astrocytes under pathophysiological conditions. Mol. Cells 2008, 25, 124-130. [PubMed]

9. Schröder, W.; Seifert, G.; Hüttmann, K.; Hinterkeuser, S.; Steinhäuser, C. AMPA receptor-mediated modulation of inward rectifier $\mathrm{K}^{+}$channels in astrocytes of mouse hippocampus. Mol. Cell. Neurosci. 2002, 19, 447-458. [CrossRef] [PubMed] 
10. Stonehouse, A.H.; Pringle, J.H.; Norman, R.I.; Stanfield, P.R.; Conley, E.C.; Brammar, W.J. Characterisation of Kir2.0 proteins in the rat cerebellum and hippocampus by polyclonal antibodies. Histochem. Cell Biol. 1999, 112, 457-465. [CrossRef] [PubMed]

11. Prüss, H.; Derst, C.; Lommel, R.; Veh, R.W. Differential distribution of individual subunits of strongly inwardly rectifying potassium channels (Kir2 family) in rat brain. Mol. Brain Res. 2005, 139, 63-79. [CrossRef] [PubMed]

12. Kofuji, P.; Biedermann, B.; Siddharthan, V.; Raap, M.; Iandiev, I.; Milenkovic, I.; Thomzig, A.; Veh, R.W.; Bringmann, A.; Reichenbach, A. Kir potassium channel subunit expression in retinal glial cells: Implications for spatial potassium buffering. Glia 2002, 39, 292-303. [CrossRef] [PubMed]

13. Hibino, H.; Inanobe, A.; Furutani, K.; Murakami, S.; Findlay, I.A.N.; Kurachi, Y. Inwardly rectifying potassium channels: Their structure, function, and physiological roles. Physiol. Rev. 2010, 90, 291-366. [CrossRef] [PubMed]

14. Ulbricht, E.; Pannicke, T.; Hollborn, M.; Raap, M.; Goczalik, I.; Iandiev, I.; Härtig, W.; Uhlmann, S.; Wiedemann, P.; Reichenbach, A.; et al. Proliferative gliosis causes mislocation and inactivation of inwardly rectifying $\mathrm{K}^{+}$(Kir) channels in rabbit retinal glial cells. Exp. Eye Res. 2008, 86, 305-313. [CrossRef] [PubMed]

15. Hibino, H.; Fujita, A.; Iwai, K.; Yamada, M.; Kurachi, Y. Differential assembly of inwardly rectifying $\mathrm{K}^{+}$ channel subunits, Kir4.1 and Kir5.1, in brain astrocytes. J. Biol. Chem. 2004, 279, 44065-44073. [CrossRef] [PubMed]

16. Ishii, M.; Fujita, A.; Iwai, K.; Kusaka, S.; Higashi, K.; Inanobe, A.; Hibino, H.; Kurachi, Y. Differential expression and distribution of Kir5.1 and Kir4.1 inwardly rectifying $\mathrm{K}^{+}$channels in retina. Am. J. Physiol. Cell Physiol. 2003, 285, C260-C267. [CrossRef] [PubMed]

17. Lichter-Konecki, U.; Mangin, J.M.; Gordish-dressman, H.; Hoffman, E.P.; Gallo, V. Gene expression profiling of astrocytes from hyperammonemic mice reveals altered pathways for water and potassium homeostasis in vivo. Glia 2008, 56, 365-377. [CrossRef] [PubMed]

18. Mulkey, D.K.; Wenker, I.C. Astrocyte chemoreceptors: Mechanisms of $\mathrm{H}^{+}$sensing by astrocytes in the retrotrapezoid nucleus and their possible contribution to respiratory drive. Exp. Physiol. 2011, 96, 400-406. [CrossRef] [PubMed]

19. Puissant, M.M.; Mouradian, G.C., Jr.; Liu, P.; Hodges, M.R. Identifying candidate genes that underlie cellular $\mathrm{pH}$ sensitivity in serotonin neurons using transcriptomics: A potential role for Kir5.1 channels. Front. Cell. Neurosci. 2017, 11, 34. [CrossRef] [PubMed]

20. Raap, M.; Biedermann, B.; Braun, P.; Milenkovic, I.; Skatchkov, S.N.; Bringmann, A.; Reichenbach, A. Diversity of Kir channel subunit mRNA expressed by retinal glial cells of the guinea-pig. Neuroreport 2002, 13, 1037-1040. [CrossRef] [PubMed]

21. Schirmer, L.; Srivastava, R.; Kalluri, S.R.; Böttinger, S.; Herwerth, M.; Carassiti, D.; Srivastava, B.; Gempt, J.; Schlegel, J.; Kuhlmann, T.; Korn, T. Differential loss of KIR4.1 immunoreactivity in multiple sclerosis lesions. Ann. Neurol. 2014, 75, 810-828. [CrossRef] [PubMed]

22. Pessia, M.; Imbrici, P.; D'Adamo, M.C.; Salvatore, L.; Tucker, S.J. Differential pH sensitivity of Kir4.1 and Kir4.2 potassium channels and their modulation by heteropolymerisation with Kir5.1. J. Physiol. 2001, 532, 359-367. [CrossRef] [PubMed]

23. Konstas, A.A.; Korbmacher, C.; Tucker, S.J. Identification of domains that control the heteromeric assembly of Kir5.1/Kir4.0 potassium channels. Am. J. Physiol. Cell Physiol. 2003, 284, C910-C917. [CrossRef] [PubMed]

24. Kofuji, P.; Ceelen, P.; Zahs, K.R.; Surbeck, L.W.; Lester, H.A.; Newman, E.A. Genetic inactivation of an inwardly rectifying potassium channel (Kir4.1 subunit) in mice: Phenotypic impact in retina. J. Neurosci. 2000, 20, 5733-5740. [CrossRef] [PubMed]

25. Greenwood, K.; Butt, A.M. Evidence that perinatal and adult NG2-glia are not conventional oligodendrocyte progenitors and do not depend on axons for their survival. Mol. Cell. Neurosci. 2003, 23, 544-558. [CrossRef]

26. Barlow, A.L.; MacLeod, A.; Noppen, S.; Sanderson, J.; Guérin, C.J. Colocalization analysis in fluorescence micrographs: Verification of a more accurate calculation of Pearson's correlation coefficient. Microsc. Microanal. 2010, 16, 710-724. [CrossRef] [PubMed]

27. Lorincz, A.; Nusser, Z. Specificity of immunoreactions: The importance of testing specificity in each method. J. Neurosci. 2008, 28, 9083-9086. [CrossRef] [PubMed]

28. Hawkins, V.; Butt, A. TASK-1 channels in oligodendrocytes: A role in ischemia mediated disruption. Neurobiol. Dis. 2013, 55, 87-94. [CrossRef] [PubMed] 
29. Neusch, C.; Rozengurt, N.; Jacobs, R.E.; Lester, H.A.; Kofuji, P. Kir4.1 potassium channel subunit is crucial for oligodendrocyte development and in vivo myelination. J. Neurosci. 2013, 21, 5429-5438. [CrossRef]

30. Bay, V.; Butt, A.M. Relationship between glial potassium regulation and axon excitability: A role for glial Kir4.1 channels. Glia 2003, 60, 651-660. [CrossRef] [PubMed]

31. Derst, C.; Karschin, C.; Wischmeyer, E.; Hirsch, J.R.; Preisig-Müller, R.; Rajan, S.; Engel, H.; Grzeschik, K.; Daut, J.; Karschin, A. Genetic and functional linkage of Kir5.1 and Kir2.1 channel subunits. FEBS Lett. 2001, 491, 305-311. [CrossRef]

32. Mi, H.; Deerinck, T.J.; Jones, M.; Ellisman, M.H.; Schwarz, T.L. Inwardly rectifying $\mathrm{K}^{+}$channels that may participate in K+ buffering are localized in microvilli of Schwann cells. J. Neurosci. 1996, 16, 2421-2429. [CrossRef] [PubMed]

33. Fakler, B.; Schultz, J.H.; Yang, J.; Schulte, U.; Brandle, U.; Zenner, H.P.; Jan, L.Y.; Ruppersberg, J.P. Identification of a titratable lysine residue that determines sensitivity of kidney potassium channels (ROMK) to intracellular pH. EMBO J. 1996, 15, 4093-4099. [PubMed]

34. Seifert, G.; Hüttmann, K.; Binder, D.K.; Hartmann, C.; Wyczynski, A.; Neusch, C.; Steinhäuser, C. Analysis of astroglial $\mathrm{K}+$ channel expression in the developing hippocampus reveals a predominant role of the Kir4.1 subunit. J. Neurosci. 2009, 29, 7474-7488. [CrossRef] [PubMed]

35. Heuser, K.; Eid, T.; Lauritzen, F.; Thoren, A.E.; Vindedal, G.F.; TaubÃll, E.; Gjerstad, L.; Spencer, D.D.; Ottersen, O.P.; Nagelhus, E.A.; et al. Loss of perivascular Kir4.1 potassium channels in the sclerotic hippocampus of patients with mesial temporal lobe epilepsy. J. Neuropathol. Exp. Neurol. 2012, 71, 814-825. [CrossRef] [PubMed]

36. Djukic, B.; Casper, K.B.; Philpot, B.D.; Chin, L.-S.; McCarthy, K.D. Conditional knock-out of Kir4.1 leads to glial membrane depolarization, inhibition of potassium and glutamate uptake, and enhanced short-term synaptic potentiation. J. Neurosci. 2007, 27, 11354-11365. [CrossRef] [PubMed]

37. Neusch, C.; Papadopoulos, N.; Muller, M.; Maletzki, I.; Winter, S.M.; Hirrlinger, J.; Handschuh, M.; Bahr, M.; Richter, D.W.; Kirchhoff, F.; et al. Lack of the Kir4.1 channel subunit abolishes $\mathrm{K}^{+}$buffering properties of astrocytes in the ventral respiratory group: Impact on extracellular $\mathrm{K}^{+}$regulation. J. Neurophysiol. 2006, 95, 1843-1852. [CrossRef] [PubMed] 